\title{
Influência da Cafeína na Resposta \\ Pressórica ao Exercício Aeróbio \\ em Sujeitos Hipertensos
}

Caffeine Influence on Blood Pressure

\section{Response to Aerobic Exercise in Hypertensive Subjects}

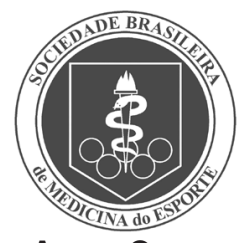

Artigo Original

Raquel Freire Cazé

Gisele Augusto Maciel Franco'

Suênia Karla Pacheco Porpino'

Alesandra Araújo de Souza

Orranette Pereira Padilhas'

Alexandre Sérgio Silva'

1. Universidade Federal da Paraíba João Pessoa, PB.

Endereço para correspondência: Alexandre Sérgio Silva

Rua Monteiro Lobato, 501, ap. 408,

Tambaú - 58039-170 - João Pessoa, PB

E-mail:ass974@yahoo.com.br

\begin{abstract}
RESUMO
A redução da pressão arterial (PA) promovida pelo exercício físico é evidente segundo a literatura atual. Mecanismos neuro-humorais explicam essa resposta hipotensora, em que a diminuição da atividade simpática apresenta-se como um dos principais mecanismos. Porém, a ingestão de alimentos ricos em cafeína (CA) pode suprimir esta atenuação simpática. O objetivo desse estudo foi elucidar o impacto da ingestão de CA na resposta pressórica ao exercício em pessoas hipertensas. Sete hipertensos (52,3 $\pm 3,3$ anos), sendo cinco mulheres, realizaram duas sessões de caminhada com 40 minutos de duração, em dois dias de treinamento, tendo previamente ingerido CA (4mg/kg de peso corporal) ou placebo (PL). A PA e a frequência cardíaca foram verificadas anteriormente a ingestão, após 15, 30, 45, 60 minutos da ingestão em estado de repouso e com 10, 20 e 30 minutos após o exercício. Os dados foram tratados por meio de estatística descritiva, e pelo teste não paramétrico de Wilcoxon ( $p<0,05)$. A média da PA aumentou de 124,9/80,9mmHg antes da ingestão de CA para 129,4/84,3mmHg 60 minutos após, ainda no repouso $(p<0,05)$. Trinta minutos após o exercício observou-se resposta hipotensora no procedimento PL (queda da PA de 122,6/79,4mmHg para 115,7/78,6mmHg), enquanto que no procedimento com CA, a PA mostrou-se significativamente mais alta em relação aos valores de repouso (aumento de 124,9/80,9mmHg para 136,9/90,9mmHg, p <0,05). Conclui-se que a ingestão de CA não só suprime a resposta hipotensora do exercício, como provoca uma hipertensão pós-exercício.
\end{abstract}

Palavras-chave: exercício físico, hipertensão, cafeína.

\begin{abstract}
Blood pressure reduction (BP) promoted by physical exercise is evident according to the current literature. Neurohumoral mechanisms explain this hypotensive response, in which decrease of the sympathetic activity appears as one of its main mechanisms. However, the ingestion of caffeine-rich food (CA) can suppress this sympathetic attenuation. The objective of this study was to elucidate the impact of CA ingestion in BP after exercise, in hypertensive individuals. Seven hypertensive subjects ( $52.3+/-3.3$ years), being 5 women, accomplished two walk sessions with 40 minutes of duration, in two days of training, having previously ingested CA (4 mg/ $\mathrm{kg}$ of body weight) or placebo (PL). BP and heart rate were verified previously to the ingestion, after 15, 30, 45, 60 minutes of the ingestion, at rest and with 10,20 and 30 minutes after exercise. Data were treated through descriptive statistics and by the non-parametric Wilcoxon test $(p<0.05)$. BP mean increased from 124.9/80.9 $\mathrm{mmHg}$ before ingestion to 129.4/84.3 mmHg 60 minutes later $(p<0.05)$. In the recovery period, after 30 minutes of exercise, hypotensive answer was observed in the PL procedure (decrease of 122.6/79.4 mmHg to 115.7/78.6 $\mathrm{mmHg}$ ), while in the procedure with CA, BP was significantly higher than at rest (increase of $124.9 / 80.9 \mathrm{mmHg}$ to $136.9 / 90.9 \mathrm{mmHg}, \mathrm{p}<0.05)$. It was concluded that CA not only suppresses the hypotensive response to exercise, but also provokes post-exercise hypertension.
\end{abstract}

Keywords: physical exercise, hypertension, caffeine.

\section{INTRODUÇÃO}

O exercício físico é considerado um forte aliado no tratamento de doenças cardiovasculares. Hipertensos que aderem a um programa de exercícios sofrem redução da pressão arterial (PA) de repouso após poucas semanas de treinamento ${ }^{(1-3)}$. Mesmo de forma aguda, nota-se uma queda da PA em comparação aos valores precedentes de repouso, fenômeno denominado hipotensão pós-exercício ${ }^{(4-6)}$. Esse efeito hipotensor prolonga-se por até 24 horas após o exercício e ocorre até mesmo em hipertensos idosos ${ }^{(5,7-10)}$.
Embora ainda não estejam totalmente esclarecidos, os mecanismos hipotensores propostos até o momento podem ser categorizados em fatores hemodinâmicos e neuro-humorais. Dentre os fatores hemodinâmicos, estão a diminuição do débito cardíaco e da resistência vascular periférica ou a combinação de ambos(6). Dentre os fatores neuro-humorais, estão o aumento de fatores de relaxamento derivados do endotélio (ERDF - óxido nítrico, prostaglandinas, EDHR), da adenosina, da sensibilidade dos barorreceptores e atenuação da atividade nervosa simpática ${ }^{(11-13)}$. Estes fatores contribuem para 
diminuição da volemia ou promovem vasodilatação, influenciando na hipotensão pós-exercício ${ }^{(6,12,14,15)}$.

Embora todos estes mecanismos hipotensores sejam ativados com o exercício, algumas atitudes cotidianas, especialmente ligadas à ingestão de alimentos, podem inibir a ação de alguns destes mecanismos vasodilatadores induzidos pelo exercício. A cafeína (CA), por exemplo, é uma substância encontrada em muitos alimentos como o café (85mg para $150 \mathrm{ml}$ ), os refrigerante de cola (até $46 \mathrm{mg}$ para $360 \mathrm{ml}$ ), os chás (30mg para $150 \mathrm{ml}$ ), e chocolates (até $20 \mathrm{mg}$ para $29 \mathrm{~g})^{(15)}$. O aumento da atividade simpática é uma evidente resposta ao uso de alimentos contendo $\mathrm{CA}^{(16)}$. Além disso, a concentração plasmática de CA, alcançada em doses entre $3 \mathrm{mg} / \mathrm{kg}$ e $6 \mathrm{mg} / \mathrm{kg}$ são suficientes para promover um efeito antagônico sobre os receptores $A_{1}$ e $A_{2 A}$ da adenosina ${ }^{(12,17)}$. Isto implica em dizer que sujeitos que realizam exercícios físicos com o objetivo de reduzir a PA, mas que ingerem produtos à base de CA, geram uma atividade concorrente entre a vasodilatação induzida pelo exercício e a vasoconstrição em resposta a ingestão destes tipos de alimentos.

Alguns estudos investigaram o efeito de CA sobre a resposta pressórica, confirmando um aumento da PA em repouso e atenuação da resposta hipotensora ao exercício ${ }^{(12,18,19)}$. No entanto, estes dados não permitem afirmar com segurança se a resposta hipotensora ao exercício é apenas atenuada, ou se a ingestão de CA provoca efeito hipertensor nos momentos pós-exercício em comparação com os valores de repouso.

Com isso, o propósito deste estudo foi determinar a influência da ingestão de CA sobre a resposta hipotensora promovida por uma sessão de exercício aeróbio em sujeitos hipertensos de meia idade.

\section{MÉTODOS}

Sujeitos do estudo: Sete sujeitos hipertensos, sendo cinco mulheres, com idade entre 48 e 58 anos (52,3 $\pm 3,3$ anos), fisicamente ativos, praticantes de caminhada com frequência mínima de três vezes por semana há pelo menos três meses, não fumantes, usuários de medicamentos anti-hipertensivos apenas no início da manhã. O projeto foi submetido ao comitê de ética em pesquisa com seres humanos do Centro de Ciências da Saúde da UFPB, sendo aprovado sob o protocolo 997/07. Todos os sujeitos assinaram o termo de consentimento livre esclarecido conforme resolução 196/96 do Conselho Nacional de Saúde.

Ingestão de cafeína e placebo: Previamente à fase do experimento, foram confeccionadas cápsulas de CA ou de um produto placebo (PL) em uma farmácia de manipulação. As cápsulas de CA foram fabricadas de acordo com o peso corporal de cada sujeito, em quantidades de $4 \mathrm{mg} / \mathrm{kg}$ de peso corporal, equivalente a aproximadamente 3,5 xícaras de café. O produto PL foi constituído por $5 \mathrm{mg}$ de veículo aerosil, um excipiente q.s.p. A ordem PL ou CA foi determinada aleatoriamente. Além disso, este procedimento foi realizado pelo modelo duplo-cego. As cápsulas foram ingeridas 60 minutos anteriormente ao início da sessão de exercício.

Exercício físico: Antes da realização do exercício, os sujeitos foram orientados a permanecerem por 72 horas em abstinência de alimentos ricos em CA ou que interfira na atividade do sistema nervoso autônomo. Foi informado aos sujeitos que os alimentos ricos em CA são o café, o pó de guaraná, refrigerantes, chás mate, chocolate. Ainda solicitou-se a abstinência da ingestão de bebidas alcoólicas neste período, assim como de qualquer alimento nas duas horas anteriores a realização do exercício.

Os sujeitos realizaram duas sessões de caminhada com duração de 40 minutos e prescrição individualizada, com intensidade entre 60\% a $80 \%$ da frequência cardíaca máxima de reserva $\left(\right.$ FCM) ${ }^{(21)}$ e intervalo de 72 horas entre cada sessão. No primeiro dia de exercício, os sujei- tos foram solicitados a manter a mesma velocidade da caminhada que utilizavam cotidianamente e tiveram suas FC registradas a cada cinco minutos. No segundo dia de exercício, o ritmo da caminhada foi determinado pela FC, de modo a manter a mesma intensidade do exercício anterior.

Medida da pressão arterial e frequência cardíaca: Ao chegarem ao local da coleta, os sujeitos foram convidados a permanecerem em repouso e sentados por 10 minutos. Em seguida, verificou-se a PA e FC de repouso. Após essas medidas foi administrada CA ou PL conforme o dia da coleta. Após a ingestão, os sujeitos foram solicitados a não realizarem qualquer esforço, tendo que permanecerem sentados por um período de 60 minutos. PA e FC foram medidas quatro vezes, a um intervalo de 15 minutos entre cada medida.

A PA foi medida na metade do treinamento. Para isto, os sujeitos eram convidados a parar o exercício e sentarem-se pelo tempo necessário para a aferição. Imediatamente após o término da caminhada os sujeitos foram solicitados a sentar, e então a PA pós-exercício foi logo verificada. Verificações adicionais foram tomadas aos 10, 20 e 30 minutos de recuperação, com os sujeitos permanecendo sentados.

A PA foi verificada pelo método auscultatório, seguindo rigorosamente o protocolo proposto nas IV diretrizes brasileiras de hipertensão arterial(21). Utilizou-se então um esfigmomanômetro aneróide da marca BD (Becton, Dickinson and Company, Franklin Lakes), com precisão de $2 \mathrm{mmHg}$, previamente calibrado contra um de coluna de mercúrio, e um estetoscópio da mesma marca.

Tratamento e análise dos dados: Os dados foram tratados por meio de estatística descritiva e foi realizado o teste não paramétrico de Wilcoxon para verificar as diferenças entre as medias de PA nas situações CA e PL. Para isto foi utilizado o software SPSS 12.0.

\section{RESULTADOS}

Resposta à ingestão de cafeína antes do exercício: como resultado da ingestão de CA, tanto a pressão arterial sistólica (PAS) quanto a pressão arterial diastólica (PAD) sofreram aumento com os sujeitos ainda em estado de repouso. O gráfico 1 apresenta o comportamento da PAS, no qual se observa que a partir de 30 minutos após a ingestão de CA, a PAS já se mostrou significativamente elevada em relação ao mesmo momento após a ingestão de PL. Esta elevação significativa da PAS se sustentou até os 60 minutos, quando se iniciou o exercício.

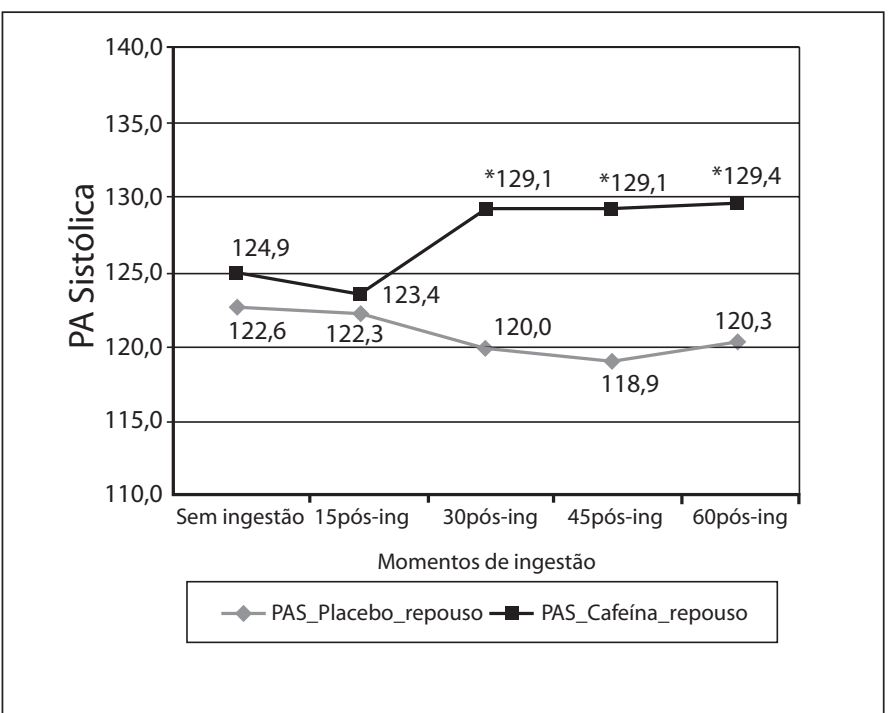

Gráfico 1. Comportamento da pressão arterial sistólica (PAS) em repouso, de acordo com o momento de ingestão de cafeína ou placebo: antes (sem ingestão) e após 15 minutos (15pós-ing), 30 minutos (30pós-ing), 45 minutos (45pós-ing), 60 minutos (60pós-ing) após a ingestão. PA = pressão arterial. Os asteriscos indicam diferença significativa para $p<0,050$. 
Um comportamento similar é mostrado no gráfico 2 para a PAD. Também, a partir dos 30 minutos, já se observou diferenças entre as PADs com PL e CA. No entanto, diferentemente da PAS, a PAD prosseguiu aumentando até os 60 minutos no procedimento com CA.

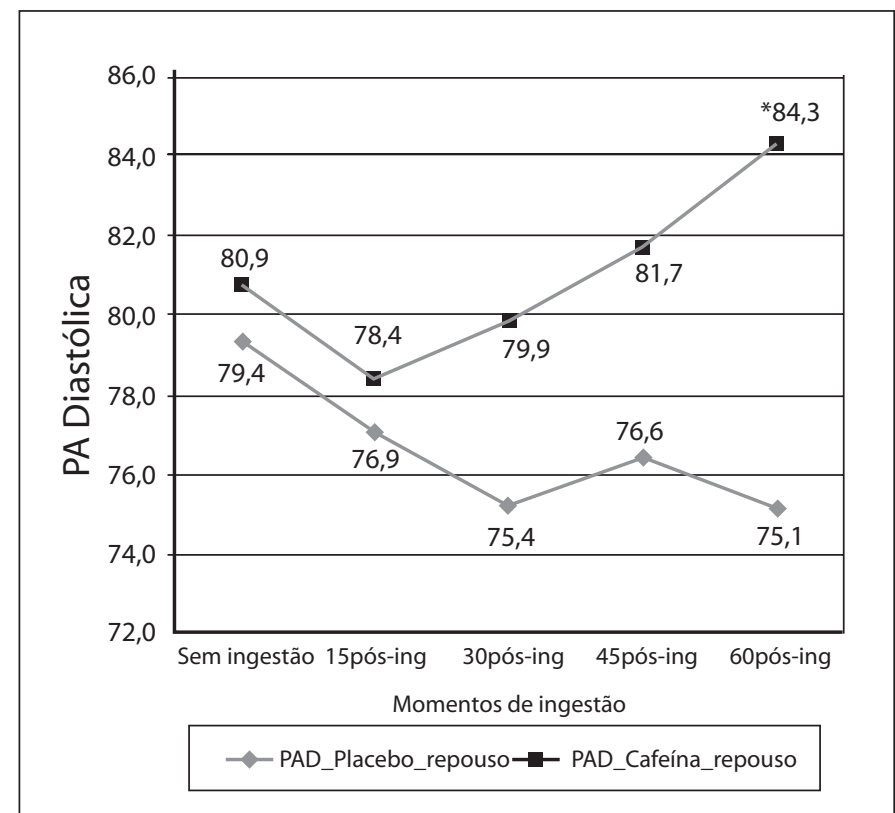

Gráfico 2. Comportamento da pressão arterial diastólica (PAD) em repouso, de acordo com o momento de ingestão de cafeína ou placebo: antes (sem ingestão) e após 15 minutos (15pós-ing), 30 minutos (30pós-ing), 45 minutos (45pós-ing), 60 minutos (60pós-ing) após a ingestão. PA = pressão arterial. Os asteriscos indicam diferença significativa para $p<0,050$

O comportamento da FC é apresentado no gráfico 3. Observase que com CA, a FC só se mostrou superior aos 60 minutos, em relação a PL. No entanto, esta diferença observada não foi estatisticamente significativa.

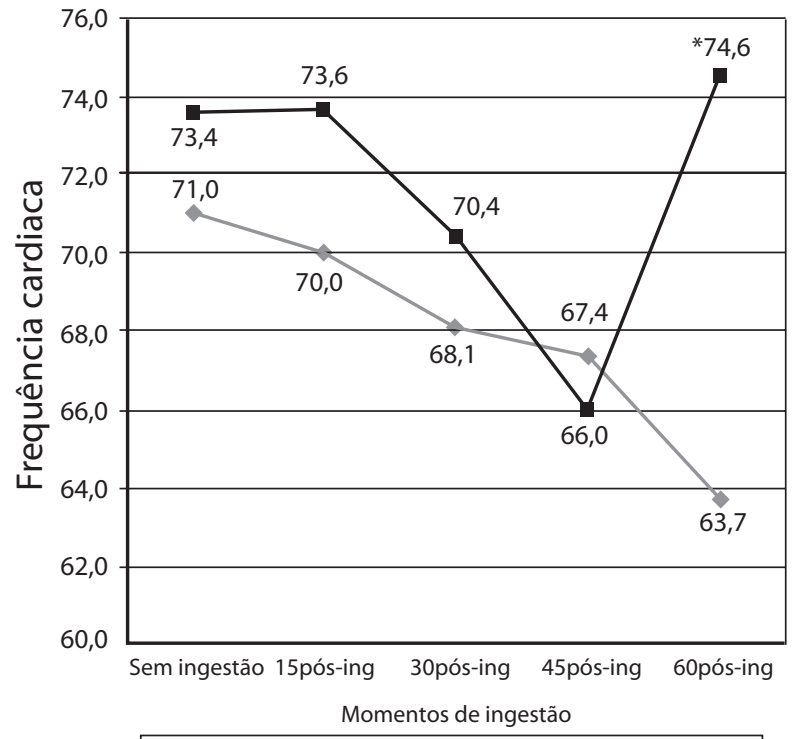

$\sim$-FC_Placebo_repouso

FC_Cafeína_repouso

Gráfico 3. Comportamento da frequência cardíaca em repouso, de acordo com o momento de ingestão de cafeína ou placebo: antes (sem ingestão) e após 15 minutos (15pós-ing), 30 minutos (30pós-ing), 45 minutos (45pós-ing), 60 minutos (60pós-ing) após a ingestão. PA = pressão arterial. Os asteriscos indicam diferença significativa para $p<0,050$
Respostas à ingestão de cafeína no comportamento pressórico pós-exercício: como resultado ao exercício físico, notou-se que CA causou um aumento tanto na PAS quanto na PAD, imediatamente após o exercício e após 30 minutos de recuperação, comparado com PL. Na medida efetuada imediatamente após o exercício, observou-se uma PAS estatisticamente superior no procedimento CA, como observado no gráfico 4. Nas medidas realizadas durante a recuperação, a PAS caiu nos primeiros 10 minutos nos dois procedimentos, mas continuou mais elevada no procedimento CA. A partir deste momento, a PAS se manteve estável em PL, mas voltou a elevar-se em CA.

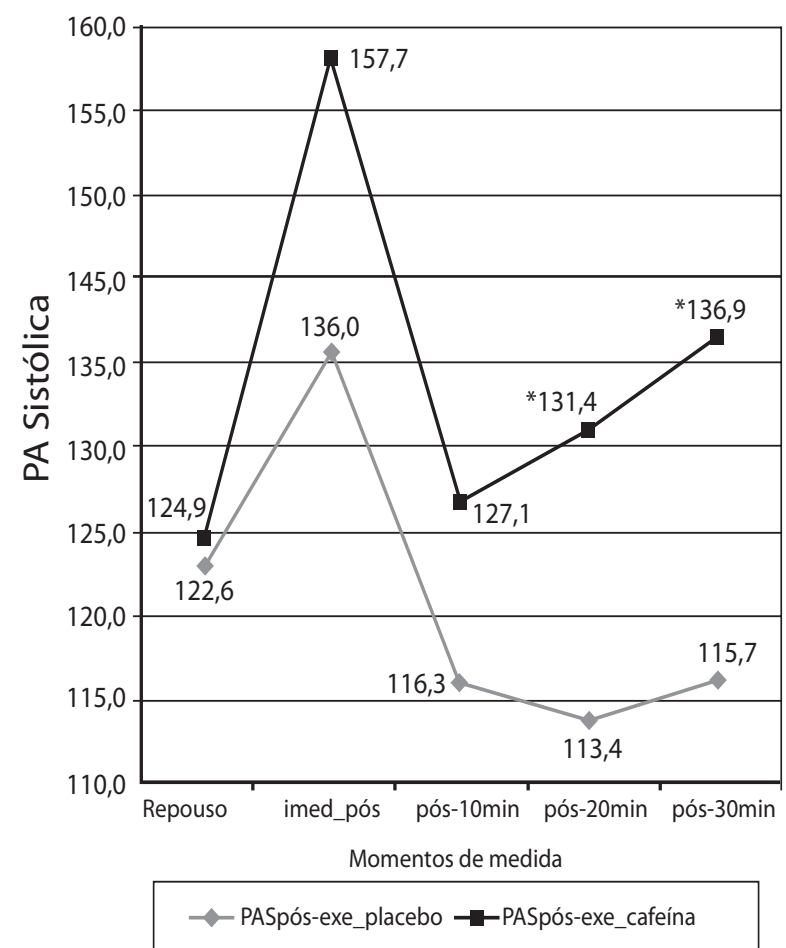

Gráfico 4. Comportamento da pressão arterial sistólica em repouso e após o exercício, com ingestão de cafeína ou placebo: em repouso, imediatamente após o exercício (imed_pós), e após 10 (pós_10min), 20 (pós_20min) e 30 (pós_30min) minutos de recuperação. Os asteriscos indicam diferença significativa para $p<0,05$.

Os valores de PAD imediatamente pós-exercício se mostraram muito similares entre os procedimentos CA e PL. No entanto, durante o período de recuperação, a PAD aumentou em CA enquanto sofreu uma discreta redução adicional em PL, (gráfico 5).

O fenômeno da hipotensão pós-exercício foi analisado comparando-se os valores da PA em repouso e aos 30 minutos após o exercício. A tabela 1 apresenta este comportamento no qual se percebe que no procedimento PL ocorreu uma redução não significativa, mas de relevância clínica tanto na PAS quanto na PAD comparado aos níveis de repouso, o que caracterizou uma leve hipotensão pós-exercício.

Enquanto isso, no procedimento CA, tanto a PAS como a PAD aumentaram significativamente em relação aos níveis de repouso. Desse modo, a ingestão de CA não somente aboliu a típica resposta hipotensora como induziu a uma hipertensão pós-exercício em relação ao estado prévio de repouso.

\section{DISCUSSÃO}

O aumento da PA a partir dos 30 minutos após a administração de CA, verificado neste estudo, indica uma atividade plasmática desta substância neste tempo decorrido desde sua administração. Este comportamento está em concordância com a literatura precedente, 


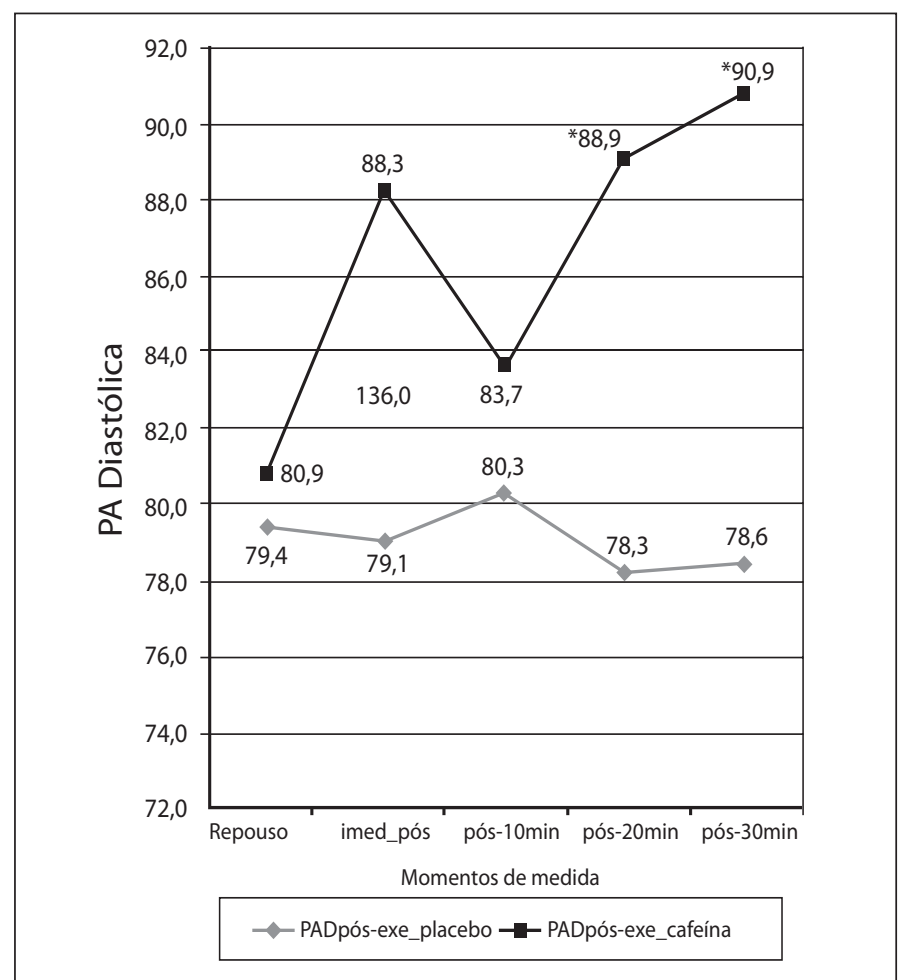

Gráfico 5. Comportamento da pressão arterial diastólica em repouso e após o exercício, com ingestão de cafeína ou placebo: em repouso, imediatamente após o exercício (imed_pós), e após 10 (pós_10min), 20 (pós_20min) e 30 (pós_30min) minutos de recuperação. Os asteriscos indicam diferença significativa para $p<0,05$.

Tabela 1. Pressões no repouso, após 30 minutos e valores de hipotensão pós- exercício (delta de pressão arterial aos 30 minutos e pressão arterial de repouso) com a ingestão de placebo ou cafeína.

\begin{tabular}{c|c|c|c}
\hline & Repouso & Após 30min exercício & HPE \\
\hline PAS placebo & $122,6 \pm 8,8$ & $115,7 \pm 12,4$ & $-6,9 \pm 13,8$ \\
\hline PAD placebo & $79,4 \pm 8,3$ & $78,6 \pm 6,1$ & $-0,9 \pm 11,6$ \\
\hline PAS cafeína & $124,9 \pm 17,4$ & $136,9^{*} \pm 17,0$ & $12,0 \pm 6,9$ \\
\hline PAD cafeína & $80,9 \pm 11,7$ & $90,9^{*} \pm 13,8$ & $10,0 \pm 7,5$ \\
\hline
\end{tabular}

Dados representam média e desvio padrăo da média. PAS = pressão arterial sistólica: PAD = pressão arteria diastólica; $\mathrm{HPE}=$ hipotensão pós-exercício. Asteriscos indicam diferenças estatísticas entre os valores de repouso versus após 30 minutos para $\mathrm{p}<0,05$

que mostra aumento da concentração plasmática de CA entre 30 e 60 minutos da administração da substância via oral(25), onde aos 60 minutos observam-se maiores concentrações de CA no organismo ${ }^{(26,}$ 27). Estes estudos indicam ainda que a diferença da administração por via oral ou intravenosa é que são necessários apenas 10 a 15 minutos para se encontrar as elevações plasmáticas pretendidas nesta segunda via de administração.

Por outro lado, embora o aumento apenas discreto na FC encontrado no presente estudo corrobore com dados precedentes ${ }^{(12)}$, outros estudos apresentaram redução cronotrópica em estado de repouso com uso de $\mathrm{CA}^{(19,28)}$. No entanto, esta redução da FC é abolida com o exercício físico ${ }^{(19)}$.

Com relação aos efeitos do exercício, o procedimento com PL resultou em hipotensão pós-exercício de 10,9mmHg para PAS e de 0,8 para PAD, em resposta à caminhada. Esta redução da PAS é considerada clinicamente importante e dentro dos limites reportados pela literatura (2 a $17 \mathrm{mmHg}$ ). Porém, os valores de PAD, se mostram bastante abaixo dos valores reportados em estudos prévios (2 a $7 \mathrm{mmHG})^{(29)}$. De qualquer maneira, a hipotensão pós-exercício foi confirmada em nosso estudo, corroborando com o consenso de que o exercício físico promove um efeito hipotensor após uma única sessão de exercício, o que ocorre tanto em normotensos quanto em hipertensos ${ }^{(5,6,11,29)}$.

No entanto, a administração de CA não somente aboliu este efeito hipotensor, como reverteu a atividade hemodinâmica para uma hipertensão pós-exercício em relação ao estado de repouso, com aumentos significativos de $12 \mathrm{mmHg}$ para PAS e $10 \mathrm{mmHg}$ para PAD. Este é um dado que deve ser encarado como muito importante, uma vez que o aumento desta natureza, tanto para a PAS quanto para a PAD, é de grande relevância clínica para hipertensos que praticam exercício físico como medida terapêutica.

Estes dados corroboram com a literatura precedente, que apontam para um aumento da PA de repouso em resposta à ingestão de CA. Porém, o dado adicional que nossos resultados trazem é a completa abolição da conhecida hipotensão pós-exercício e ainda uma substituição deste efeito por uma resposta hipertensiva após a realização de uma sessão de exercício físico.

Os mecanismos explicadores para este fenômeno parecem estar ligados a uma vasoconstrição provocada por aumento da atividade simpática e ação da CA sobre a adenosina. Estes mecanismos ainda não estão totalmente esclarecidos, mas sabe-se que a CA é uma substância estimulante do sistema nervoso central (SNC) e sua ação na resistência vascular periférica é mais fraca que o seu efeito no SNC(15,30).

Quanto à ação sobre a adenosina a CA causa um efeito bloqueador dos receptores $A_{1}$ da adenosina, aumentando a liberação de substâncias vasoconstrictoras e bloqueando os receptores $A_{2 A}$ da adenosina ${ }^{(17)}$. Estes efeitos combinados levam a uma atividade vasoconstrictora, com conseqüente aumento da $\mathrm{PA}^{(28,29)}$.

A importância dos dados deste estudo para a população reside no fato de que CA é muito consumida por meio de vários alimentos, como o café, os refrigerantes de cola, os chás e chocolates ${ }^{(15)}$. A quantidade de CA utilizada no presente estudo corresponde a três xícaras e meia de café (150ml para cada xícara). Esta é uma quantidade que pode ser razoavelmente consumida por muitas pessoas em pequeno espaço de tempo. Ainda vale salientar que algumas pessoas usam substâncias emagrecedoras que são adrenérgicas. Da mesma forma que a CA, essas substâncias podem estar fazendo um efeito concorrente com a hipotensão pós-exercício.

Dado a este fenômeno, é importante que investigações futuras quantifiquem a prevalência da ingestão destes alimentos (em termos de frequência e quantidades) e posteriormente analisem os efeitos diretos deste consumo sobre as respostas hipotensoras do exercício físico.

\section{CONCLUSÃO}

Os dados do presente estudo demonstraram que concentrações de $4 \mathrm{mg} / \mathrm{kg}$ de cafeína promovem hipertensão após uma sessão de exercício aeróbio. Este dado tem uma importante implicação clínica, em se considerando que a resposta normal da PA ao exercício é normalmente hipotensora. Indica, portanto, que o consumo de alimentos ricos em CA pode estar causando uma atividade concorrente com a resposta hipotensora que o exercício ocasiona. Por isso, sugere-se o desenvolvimento de mais estudos nesta área para criar um corpo de evidências sobre o assunto. Além disso, deve-se avaliar o consumo de produtos ricos em CA antes e após sessões de exercícios.

Todos os autores declararam não haver qualquer potencial conflito de interesses referente a este artigo. 


\section{REFERÊNCIAS}

1. Simonetti LA. Resposta hipotensora de hipertensos essenciais a protocolo supervisionado de exercícios aeróbios de longa duração. [Tese de Doutorado] São Paulo: Universidade de São Paulo; 2002.

2. Pinto VL, Meirelles LR, Farinatti PTV. Influência de programas não-formais de exercícios (doméstico e comunitário) sobre a aptidão física, pressão arterial e variáveis bioquímicas em pacientes hipertensos. Rev Bras Med Esporte. 2003;9(5):99-104.

3. Oliveira RB, Vianne LC, Ricardo DJ, Castro CLB, Araújo CGS. Dez anos de programa de exercício supervisionado: características dos pacientes e das intecorrências clínicas. Socerj. 2006;19(2):131-7.

4. Pescatello LS, Franklin BA, Fagard R, Farquhar RWB, Kelley GA, RAY CA. Exercise and hypertension. Med Sci Sports Exerc. 2004;36(3)533-53.

5. Brandão-Rondon MUP, Alves MJ, Braga AM, Teixeira OT, Barretto AC, Krieger EM, et al. Post-exercise blood pressure reduction in elderly hypertensive patients. J Am Coll Cardiol. 2002;39(4):676-82.

Brum PC, Brandão-Rondon MUP, Silva GJJ, Krieger EM. Hipertensão Arterial e Exercício Físico Aeróbio. In Cardiologia do exercício: do atleta ao cardiopata. 2nd ed. Barueri- SP:Manole; 2005. p.167-75.

6. Kokkinos PF, Papademetriou V. Exercise and hypertension. Coron Artery Dis. 2000;11:99-102.

7. Forjaz $C L$, Tinucci $T$, Ortega $K C$, Santaella DF, Mion D Jr, Negrão CE. Factor affecting post-exercise hypotensive humans. Blood Press Monit. 2000;5(5-6):255-62.

8. Thompson PD. O Exercício e a Cardiologia do Esporte. 3 ed. Barueri-SP: Manole; 2004. p.91-103.

9. Baster T, Baster-Brooks C. Exercise and hypertension. Aust Fam Physician. 2005;34(6):419-24.

10. Halliwill JR. Mechanisms and clinical implications of post-exercise hypotension in humans. Exercise and Sports Sci Rev. 2001;29:65-75.

11. Notarius CF, Morris BL, Floras JS. Caffeine attenuates early post-exercise hypotension in middle-aged subjects. Am J Hypertens. 2006;19:184-8.

12. Zago AS, Zanesco A. Óxido Nítrico, Doenças Cardiovasculares e Exercício Físico. Arq Bras Cardiol. 2006;87(6):264-70.

13. Cononie CC, Graves JE, Pollock ML, Phillips ML, Summers C, Hagberg JM. Effect of Exercise Training on Blood preassure in 70-79 year old on men and women. Med Sci Sports Exerc. 1991;23:505-11.

14. Tirapegui J. Nutrição, metabolismo e suplementação na atividade física. São Paulo-SP: Atheneu; 2005. p.181-7.

15. Notarius CF, Atchison DJ, Rongen GA, Floras JS. Effect of adenosine receptor blockade with caffei- ne on sympathetic response to handgrip exercise in heart failure. Am J Physil Heart Circ Physiol. 2001;281:H1312-18.

16. Smits P, Thien T, Laar AV. Circulatory effects of coffee in relation to the pharmacokinetics of caffeine. Am J Cardiol. 1985;56:958-63.

17. Sung BH, Lovallo WR, Whitsett T, Wilson MF. Caffeine elevates blood pressure response to exercise in mild hypertensive men. Am J Hypertens. 1995;8(12 Pt 1):1184-8.

18. Daniels JW, Molé PA, Shaffrath JD, Stebbins CL. Effects of caffeine on blood pressure, heart rate, and forearm blood flow during dynamic leg exercise. J Appl Physiol. 1998;85(1):154-9.

19. Karvonen MJ, Kentala E, Mustala O. The effects of training on heart rate: a longitudinal study. Ann Med Exper Fenn. 1957;35(3);307-15.

20. Sociedade Brasileira de Hipertensão, Sociedade Brasileira de Cardiologia, Sociedade Brasileira de Nefrologia. IV Diretrizes Brasileiras de Hipertensão Arterial. São Paulo: BG Cultural; 2002.

21. Sinclair CJD, Geiger JD. Caffeine use in sports. A pharmacological review. J Sports Med Phys Fitness. 2000;40(1):71-9.

22. McLean C, Graham TE. The impact of gender and exercise on caffeine pharmacokinetics. Med Sci Sports Exerc. 1998;30(Suppl5):S243-6.

23. Graham TE. Caffeine, coffee and ephedrine: impact on exercise performance and metabolism. J. Appl. Physiol. 2001;26(Suppl1):S103-19.

24. Sung BH, Lovallo WR, Pincomb GA, Wilson MF. Effects of caffeine on blood pressure response during exercise in normotensive healthy young men. Am J Cardiol. 1990;65(13):909-13.

25. Santaella DF, Campos MRN, Araújo EA, Exercício e relaxamento alteram a modulação autonômica da hipotensão pós-exercício diferentemente em normotensos e hipertensos. Hipertensão. 2003;6(Supl36):83.

26. Forjaz CLM, Brandão-Rondon MUP, Negrão CE. Efeitos hipotensores e simpatolíticos do exercício aeróbio na hipertensão arterial. Rev Bras Hipertens. 2005;12(4):245-50.

27. Cavalcante JWS, Santos Jr. PRM, Menezes MGF, Marques HO, Cavalcante LP, Pacheco WS. Influence of Caffeine on Blood Pressure and Platelet Aggregation. Arq Bras Cardiol. 2000;75(2):102-5.

28. Corti R, Binggeli C, Sudano I, Spieker L, Hänseler E, Ruschitzka F, Chaplin WF, Lüscher TF, Noll G. Coffee acutely increases sympathetic nerve activity and blood pressure independently of caffeine content: role of habitual versus nonhabitual drinking. Circulation 2002:106: 2935-40. 